\title{
Response training in young children's discrimination shift learning
}

\author{
MARILYN NOVAK and STUART I. OFFENBACH \\ Purdue University, West Lafayette, Indiana
}

\begin{abstract}
A passive responding procedure, pointing at the correct stimulus, was used to determine whether preschool children would test hypotheses from the relevant attribute in a discrimination shift task. Some children were administered the initial task in the usual manner, with positive or negative feedback. About half of these children did not reach criterion. Other children were told which stimulus to point at. These children were then given positive feedback, but were not told why the response was correct. All children then received a reversal or a nonreversal shift. The children who were told which stimulus to choose learned both shifts in the same manner that the children who attained criterion on their own did. The children who did not attain criterion, who failed to learn, performed on a nonreversal shift like the learners did on the reversal (the nonlearners' reversal performance was like the learners' nonreversal performance). The results show that a simple directed response can limit the sample of hypotheses that young children test in discrimination learning even when the purpose of the response is ambiguous.
\end{abstract}

The discrimination transfer paradigm (in its several forms) has been a rich source of information regarding the developmental aspects of how children learn. The results from the many experiments that have used these tasks also have been used to support a number of theoretical positions in developmental psychology (e.g., H. H. Kendler \& T. S. Kendler, 1962; Spiker \& Cantor, 1973; Tighe \& Tighe, 1972; Zeaman \& House, 1974). Generally, the shift paradigm consists of a pair of two-choice discrimination learning tasks. In each task, one value of a more general attribute (e.g., red colors or square shapes) is perfectly correlated with positive feedback (is relevant), and another value of these attributes is randomly associated with feedback (is irrelevant). The relationship between the relevant values of the first and second tasks determines the ease or difficulty with which that second "shift" task is acquired. For example, a traditional reversal shift task might use the color red as the relevant cue in original learning and the color blue as the relevant cue in the second task (two shape values would be irrelevant in both tasks). If, on the other hand, one of the shapes had become relevant in the second task (and colors were made irrelevant), a nonreversal shift would be required of the learner. Developmentally, it has been reported by some investigators that children younger than 5 years have more difficulty in learning a reversal shift than they do a nonreversal shift. The opposite pattern has been reported for older children (see Esposito, 1975, and Wolff, 1967,

This study is based on a master's thesis conducted by the first author under the direction of the second author and submitted to Purdue University in partial fulfillment of the requirments for the Master of Science degree. We thank the Committee members, Larry M. Raskin and Gerald E. Gruen, for their assistance in this work. M. Novak is now at the Department of Social Services of the State of Michigan. Requests for reprints should be addressed to Stuart I. Offenbach, Department of Psychological Sciences, Purdue University, West Lafayette, IN 47907. for reviews). The developmental pattern of results, when it occurs, has been interpreted by the Kendlers $(\mathrm{H}$. $\mathrm{H}$. Kendler \& T. S. Kendler, 1962; T. S. Kendler \& H. H. Kendler, 1970) and others (e.g., Spiker \& Cantor, 1973) to indicate that young children learn via single-unit response associations between the relevant cue (red) and the correct response, whereas older children respond to the abstract qualities of the cue or the class to which the cue belongs (e.g., color). Thus, on the reversal shift, young children must relearn which cues are positive and which are negative, whereas older children still can respond to "color," changing only the instrumental response (choosing red to choosing blue). Early on, T. S. Kendler and H. H. Kendler (1959) found that 5-year-old children appeared to be at a transition point. Some children learned the initial task slowly (single-unit learners) and a nonreversal easily. Other children (mediators) learned the initial task quickly and had an easier time with the reversal shift.

Another group of theorists (e.g., Tighe \& Tighe, 1972; Zeaman \& House, 1974) have argued that these developmental patterns reflect differences in attention rather than differences in kind of learning. In these approaches, attention appears to refer to a selective stimulus-processing function whereby one cue (or attribute or dimension) is processed at a time (and others are ignored or not processed until later). One line of support for the attention position has been the finding that discrimination shift learning is determined by "dimensional preferences" (which seems to refer to a preexperimental, possibly arbitrary, bias to attend to one specific dimension). Overall, any procedure that directs or limits attention to the relevant cue should lead to faster acquisition of a reversal shift, whereas a procedure that keeps attention on the irrelevant dimension(s) would facilitate nonreversal shifts. 
Recently, other approaches have joined the growing list of theoretical considerations of the development of learning. Most notably, hypothesis testing theory (see Levine, 1975) has been extended to account for many aspects of development by Gholson and his colleagues (see Gholson, 1980 , or Gholson \& Beilin, 1980, for a review). One focus of hypothesis theory has been to describe response selection strategies or "systems" (Gholson's term: Gholson, Levine, \& Phillips, 1972) that children use to choose which hypotheses to test on a given trial. Although the hypothesis theory has received support from studies of children's discrimination learning, it has not been applied directly to the transfer paradigm.

In comparing these approaches, several differences stand out. One important one concerns whether acquisition depends on the manner in which the child organizes the stimuli and the task (hypothesis theory) or whether what is learned is structured primarily by what the task demands (attention theory and mediation theory). Hypothesis theory requires that the learner actively select and structure the task, whereas attention and mediation theory adopt a more passive attitude relying on characteristics of the stimuli (Aschkenasy \& Odom's, 1982, discussion of distinctiveness-based and predisposed salience is an example). One implication of an approach that relies on children's noticing the relevant cues via attention is that a procedure based on experimenter-directed passive responses might not produce attention to the relevant cue, thus reducing or eliminating learning. Since the hypothesis testing position requires active selection and testing regardless of procedural differences of this type, a passive response procedure should not interfere with knowledge of the correct hypothesis.

The present experiment was a test of this possibility. Passive responding during learning was accomplished by telling children which stimulus to point to on each trial of a discrimination task, but not why to choose it. Pointing represents a "strategy" for responding, but not necessarily a strategy for selecting which response to make. However, if hypothesis testing occurs as a result of internal organization, the pointing response should lead children to test hypotheses about which cue is correct on each trial. Given that children can process information about hypotheses following positive feedback on these trials (as do adults; see Levison \& Restle, 1973), the appropriate hypothesis should be educed. This would lead to rapid reversal learning and slower nonreversal learning. This learning pattern also would be expected for children who were trained in the usual transfer-task procedure (making whichever response they wanted to), provided they tested the correct hypothesis and attained criterion. Children who for whatever reason stay with an incorrect hypothesis or never test the correct hypothesis probably would have more difficulty with a reversal task.

\section{METHOD}

\section{Subjects}

One hundred and nine preschool children, with a mean age of 58 months (range 44 to 66 months) participated in the study. The children were from a day-care center adjacent to the Purdue University campus. The distribution of boys and girls in each experimental condition was approximately equal.

\section{Procedure}

The design of the experiment was a $3 \times 2$ factorial, with three learning groups (learners who were children who attained the criterion on the initial learning task; nonlearners who failed to attain the initial learning-task criterion; and children in the "response instructed" group who were told which stimulus to choose) and two shift conditions (reversal and nonreversal).

The learning-task stimuli varied on two nonspatial attributes: size (ratio of 2:1) and color (orange and green). The stimulus pairs were constructed of colored papers mounted on $5 \times 8$ in. blank cards. The children were tested, one at a time, in a quiet room adjacent to the play area. The instructions informed the children that a "guessing" game would be played, that the correct object was to be selected each time by pointing to it, that the object could be correct because of its size (large or small) or its color (orange or green), and that the experimenter would tell the child whether the choice was correct or incorrect. In addition to the verbal feedback, the child was given a marble (which was exchanged for a prize following the session) after each correct response.

Both the original and shift tasks were presented for 48 trials or until a criterion of 10 consecutive correct responses had been attained. The children in the training condition were given the following additional instructions: "I will help you with some cards (the first stimulus card was shown). Put your finger on this one (the experimenter pointed to one of the stimuli)." When the child pointed, the experimenter said "correct" and dispensed a marble. At no time during the task did the experimenter identify why the choice of that cue was correct. The reversal or nonreversal shift task was administered immediately following the criterion run (or after 48 trials). In the reversal shift, the incorrect cue of the relevant attribute in original learning was now correct (e.g., a shift from large to small or from orange to green). For the nonreversal shift, a cue from the previously irrelevant atrribute became relevant (e.g., a shift from large to green or orange, or a shift from green to large or small). The only change in the procedure was that the children in the response training condition were told "I helped you with those cards, but you can do these by yourself. Remember, point to the thing on the card that you think is right."

\section{RESULTS}

\section{Original Learning}

Seventy-two of the 109 participants were administered the initial learning task under standard learning conditions. Thirty-one of these children learned, whereas 41 failed to attain the criterion. The remaining 37 children were administered the response training procedure. There were no significant age differences among these groups of children.

The only data from initial learning that were analyzed in detail were the error scores of those children who attained the criterion. The children who were later given the reversal shift did not differ from those children who later had the nonreversal shift. Similar comparisons for the nonlearners and the response-trained group were not meaningful, because there was little or no variability in either group.

\section{Shift Task Performance}

The mean number of errors to criterion for each group is shown in Table 1. A repeated-measures analysis of variance of the number of errors made in each block of eight trials yielded three significant interactions: shift $\times$ initial training $[F(2,103)=4.75]$, shift $\times$ trials $[F(3,309)$ 
Table 1

Mean Number of Errors to Criterion on the Shift Task

\begin{tabular}{lccccc}
\hline & \multicolumn{2}{c}{ Reversal } & & \multicolumn{2}{c}{ Nonreversal } \\
\cline { 2 - 3 } \multicolumn{1}{c}{ Group } & Errors & $\mathrm{n}$ & & Errors & $\mathrm{n}$ \\
\hline Criterion-Trained Learners & 13.7 & 17 & & 22.4 & 14 \\
Criterion-Trained Nonlearners & 19.8 & 20 & & 13.5 & 21 \\
Passive Responders & 14.7 & 19 & & 18.1 & 18 \\
\hline
\end{tabular}

Note $-n=$ number of children in each condition.

$=4.71]$, and the three-way interaction of shift type, initial training, and trials $[\mathrm{F}(6,309)=5.41]$ (all $\mathrm{p}<.05)$. In addition, there was a significant trials main effect $[F(3,132)=6.27]$. Inspection of the data (Table 1) indicated that the children receiving the response training performed similarly to those who independently attained criterion on the initial learning task. In addition, the children who had a criterion run, whether spontaneously or through the sequence of 10 experimenter-directed responses, learned the reversal shift more rapidly than those children who did not have a criterion run.

\section{DISCUSSION}

The results support the conclusion that children tested hypotheses and learned about the relevant attribute even when their learning-task responses were passive and directed by the experimenter. Possibly, the

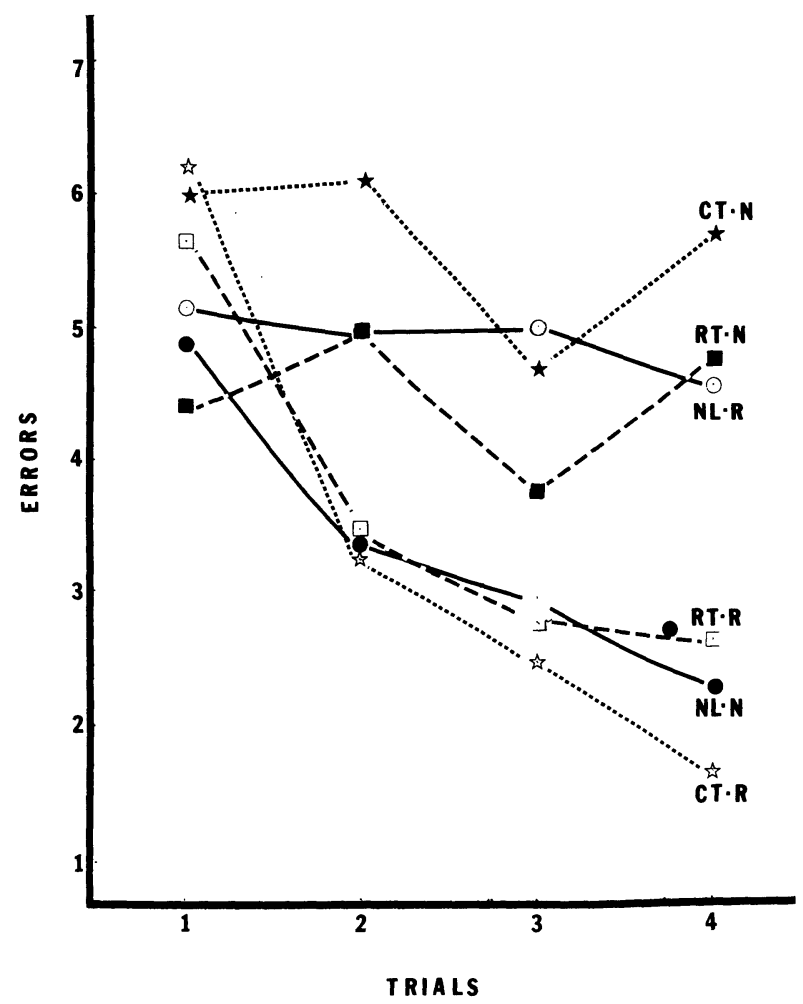

Figure 1. Mean number of errors in each block of eight trials of the reversal $(R)$ and nonreversal $(N)$ shift task for the criteriontrained (CT) learners, the nonlearners (NL), and the response-trained (RT) children. training procedure forced the preschool-aged children in this study to limit their focus, or attention, to the hypotheses from one attribute. This apparently occurred even though the response was entirely passive. These children were not told which attribute was relevant. And the experimenter did not tell them, for example, that "the red one was correct." Rather, the experimenter simply said "correct" after the child had pointed to the stimulus. One effect of such limited hypothesis testing might be that children would learn little about other attributes, causing them to perform poorly on the nonreversal shift. A similar pattern might be observed among the children who were administered the task in the usual manner. However, these children probably tested hypotheses from more than one attribute. Those who did attain criterion obviously tested hypotheses from the relevant attribute, whereas those who did not attain criterion probably failed to select the relevant hypothesis. The fact that the 4-year-old children's response-directed original learning led to performance on the shift task that mimicked exactly the performance of the children who responded spontaneously (on their own) strongly implies that even a mild externally imposed strategy (pointing) can lead to efficient learning-task performance and transfer.

There also was evidence that children who did not attain criterion (the nonlearners) tested hypotheses, perhaps exclusively, from the irrelevant attribute. Their performance on the nonreversal shift was almost identical with the reversal shift performance of the children who attained criterion (Figure 1). That pattern was expected. By the end of the initial learning task, most of the children would have eliminated all but one or two hypotheses from further consideration. If these few remaining hypotheses were relevant to the solution of the shift task, the children should have attained the criterion ( $76 \%$ of them did). However, when the hypotheses that were being used at the end of the initial task were not relevant to the shift-task solution, few children (6 to 34) should have learned the shift discrimination.

One limitation of this study is that we could not differentiate between the proposition that the available subset of hypotheses was limited in some way and the proposition that children's attention was somehow limited or selective. The results do, however, support a sort of compromise, namely, that children's response choices are limited in some manner and that very simple "strategies" can be used to manipulate that set of choices (regardless of whether the choices are determined by hypothesis testing or by attention). Determining how such simple strategies can be efficiently employed in other experimental tasks should enable the investigator to better control the learning environment.

\section{REFERENCES}

Aschkenasy, J. R., \& ODOM, R. D. (1982). Classification and perceptual development: Exploring issues about integrality and differential sensitivity. Journal of Experimental Child Psychology, 34, 435-448.

Esposito, N. J. (1975). Review of discrimination shift learning in young children. Psychological Bulletin, 82, 432-455.

GHolson, B. (1980). The cognitive-developmental basis of human learning: Studies in hypothesis testing. New York: Academic Press.

Gholson, B., \& BeILIN, H. (1980). A developmental model of human learning. In H. W. Reese \& L. P. Lipsitt (Eds.), Advances in child development and behavior (Vol. 13, pp. 47-81). New York: Academic Press.

Gholson, B., Levine, M., \& Phillips, S. (1972). Hypotheses, strategies, and stereotypes in discrimination learning. Journal of Experimental Child Psychology, 13, 423-446.

Kendler, H. H., \& Kendler, T. S. (1962). Vertical and horizontal processes in problem solving. Psychological Review, 69, 1-16.

KendleR, T. S., \& KendLER, H. H. (1959). Reversal and non-reversal shifts in kindergarten children. Journal of Experimental Psychology, 58, 56-60.

Kendler, T. S., \& Kendler, H. H. (1970). An ontogeny of optional shift behavior. Child Development, 41, 1-27.

LEVINE, M. (1975). A cognitive theory of learning research on hypothesis testing. Hillsdale, NJ: Erlbaum.

LEVISON, M. J., \& RESTLE, F. (1973). Effects of blank-trial probes on concept-identification problems with redundant relevant cue solutions. Journal of Experimental Psychology, 98, 368-374.

SPIKER, C. C., \& CANTOR, J. H. (1973). Applications of Hull-Spence 
theory to the transfer of discrimination learning in children. In $\mathrm{H}$. W. Reese (Ed.), Advances in child development and behavior (Vol. 8, pp. 224-288). New York: Academic Press.

Tighe, L. S., \& Tighe, T. J. (1972). Stimulus control in children's learning. In A. D. Pick (Ed.), Minnesota Symposia on Child Development (Vol. 6, pp. 128-157). Minneapolis: University of Minnesota Press. WoLfF, J. L. (1967). Concept-shift and discrimination-reversal learning in humans. Psychological Bulletin, 68, 369-408.
Zeaman, D., \& HousE, B. J. (1974). Interpretations of developmental trends in discriminative transfer effects. In A. D. Pick (Ed.), Minnesota Symposia on Child Development (Vol. 8, pp. 144-186). Minneapolis: University of Minnesota Press.

(Manuscript received for publication September 24, 1984.) 